\title{
The last polymath
}

\section{Henning Schmidgen praises a tome on Helmholtz, titan of nineteenth-century science.}

$\mathrm{H}$ ermann von Helmholtz is a towering figure in the history of modern science. In the nineteenth century, his peers in experimental physiology and physics regarded him as a scientific genius, and the German public nicknamed him 'Imperial Chancellor of the Sciences', given his multifaceted activities as a manager of scientific institutions. These ranged from his own laboratories in Heidelberg and Berlin to the Imperial Physical Technical Institute, established in Berlin with financial support from a friend, industrialist Werner von Siemens. Moreover, in contrast to Charles Darwin or Louis Pasteur (or, later, Albert Einstein), Helmholtz's pathbreaking achievements were not confined to one discipline.

Against this background, historians and philosophers consider Helmholtz to be one of the last - if not the last - polymaths in the history of science. His is a legacy so rich it is difficult to grasp.

Born in 1821, Helmholtz trained as a physician at the University of Berlin. In the 1840 s and 1850 s, he devised the ophthalmoscope - an instrument for examining the inside of the eye - and took innovative measurements of the speed of nerve impulses. He performed extensive experiments in sensory physiology, resulting in multi-volume manuals on optics and acoustics, published during the 1860s. He then established himself as a physicist. During the 1870s and 1880s, his main fields of research were electrodynamics and thermodynamics. By the time he died, in 1894, Helmholtz had gained further recognition as a philosopher of science and nature in the mould of Immanuel Kant, advocating precision work in the laboratory alongside sophisticated theory.

Historian David Cahan copes confidently with these complexities in his monumental new biography. The result of almost three decades of scholarly work, Helmholtz is a comprehensive and timely account. In recent years, historians of science have published several studies devoted to isolated aspects of Helmholtz's work - his contributions to neurophysiology and hydrodynamics, as well as his epistemology and aesthetics.

However, anyone interested in the complete picture was forced to go back more than 100 years, to a bulky biography by German mathematician Leo Königsberger. In no fewer than three volumes published in 1902-03, Königsberger, a friend of the Helmholtz family, depicted the German

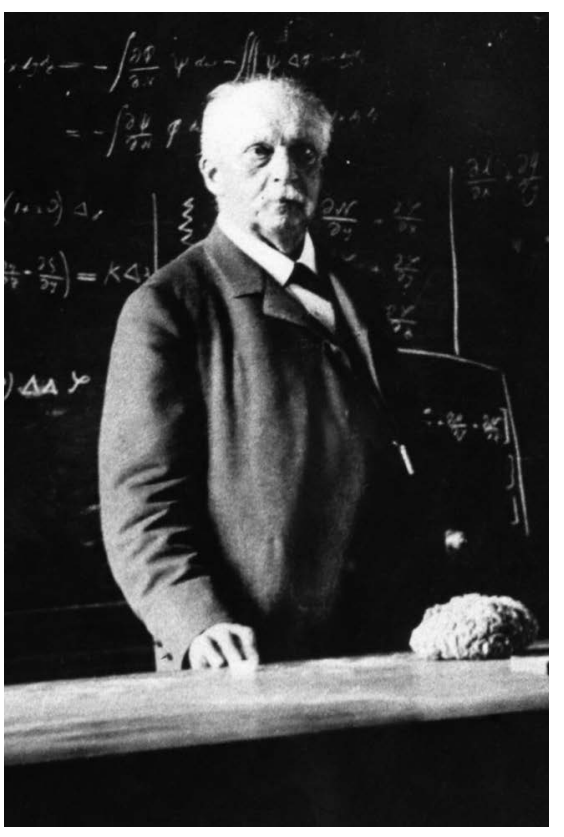

Hermann von Helmholtz in 1894.

scholar as a lonesome genius whose scientific success was the result of unusual giftedness combined with extremely hard work.

Helmholtz is an impressive corrective to such partial or simplistic treatments. The book not only accounts for the German scholar's voluminous publications (his Lectures on Theoretical Physics alone comprise six volumes, posthumously published between 1897 and 1907), but also encompasses an uncounted number of manuscripts and letters dispersed in archives all over the world. The result is as compelling as it is convincing.

Cahan's Helmholtz emerges as a liberal conservative devoted to his family, and as a talented and ambitious scientist whose career unfolded in close connection with the culture and society of Imperial Germany. This is not to downplay the significance of Helmholtz's achievements.

On the contrary, Cahan aims to illuminate these achievements - their texture, as it were. He ranges over Helmholtz's work: the introduction of innovative instruments and experimental techniques; extensive writing, often aimed at securing priority for his discoveries and inventions; and intense communication with scientists, engineers and politicians in Germany and abroad. Helmholtz, we learn, was a skilled networker and salesperson of all things scientific, as well as an exceptional researcher.

Drawing on recent approaches to the
Helmholtz: A Life

in Science

DAVID CAHAN

University of Chicago

Press (2018)

history of science that highlight cultural and social context, Helmholtz reveals the preconditions for the successful reception and propagation of Helmholtz's work. It becomes clear that, in the late 1840 s, Helmholtz referred to and relied on German telegraph technology when performing his precision measurements of nerve impulses. Cahan also shows that his organizational achievements as 'Imperial Chancellor of the Sciences' would not have been possible without the political and administrative network that he and his colleague Emil du Bois-Reymond began to weave at the start of their careers, in Berlin. Readers of Cahan's wonderful book will even be convinced that Helmholtz's laboratory work on physiological optics and acoustics became popular among artists, because he met painters such as Adolph von Menzel, and musical luminaries including Richard Wagner.

Cahan effortlessly depicts and analyses Helmholtz's trajectory as a movement through various disciplinary, institutional and cultural contexts. His account is not a simple biography or a mere retracing of events. As the subtitle indicates, its ambitious goal is to explore the relationships between life and science.

The guiding thread of this exploration is what Cahan calls the "civilizing power" of science. For Helmholtz, science was neither blunt profession nor pure vocation; it was a way of life. Truth was the ultimate goal, reached through the crucial means and methods of empirical care, precision and the reproducibility of findings. Science in the Helmholtzian sense is vitally important not just for individuals, but also for the society that they belong to.

Cahan demonstrates that Helmholtz strikingly embodies the cognitive and ethical potential of science. His book is the new standard for all Helmholtz scholars, a comprehensive guide and crucial resource for historians, philosophers or sociologists interested in this scientific luminary. Helmholtz is also a highly topical book for those impressed by and convinced of the social significance of science.

Henning Schmidgen is a historian of science and professor of media studies at Bauhaus University in Weimar, Germany. He is the author of The Helmholtz Curves. e-mail:henning.schmidgen@uni-weimar.de 